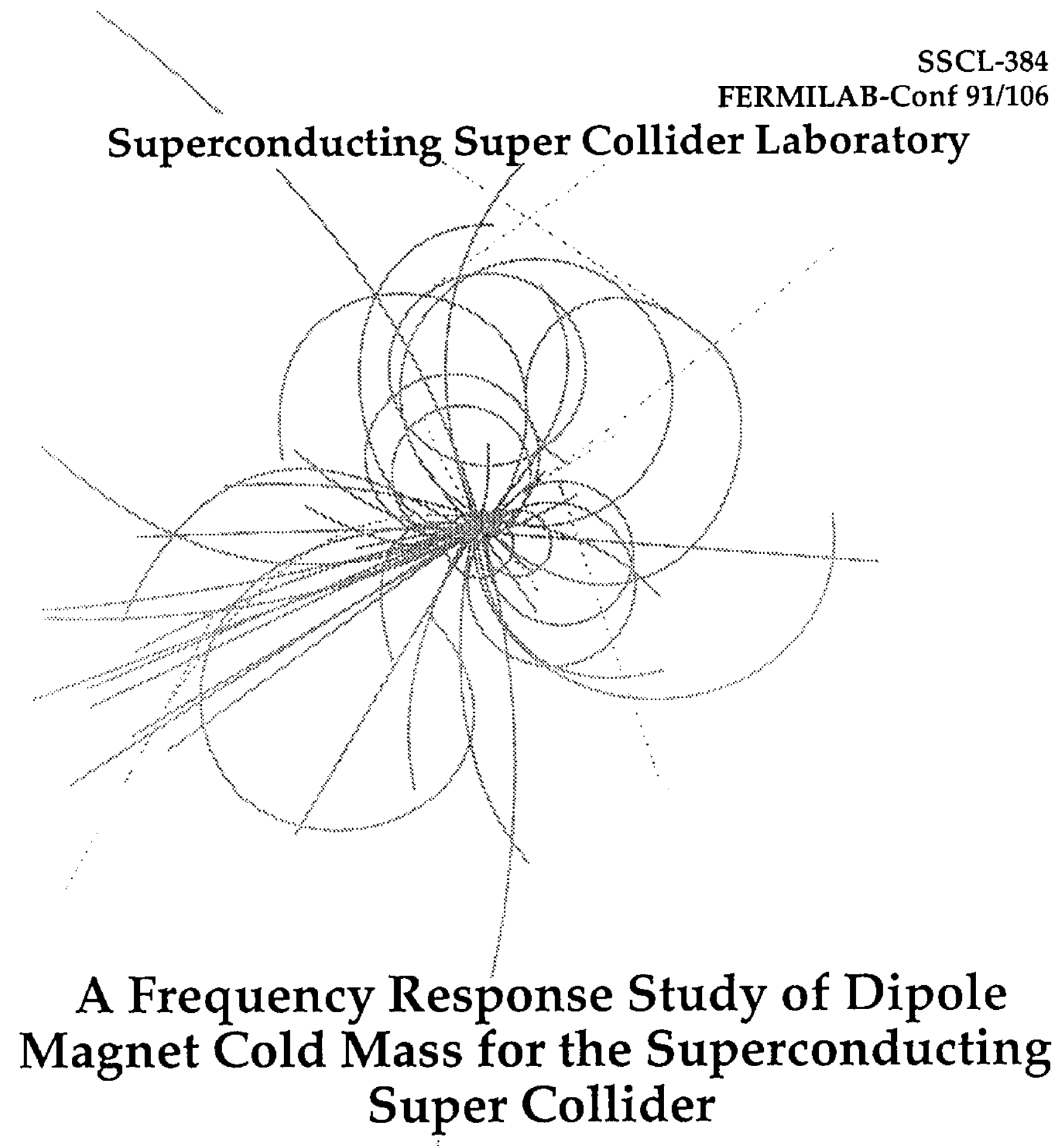

K. K. Leung and T. Nicol

March 1991 


\title{
A Frequency Response Study of Dipole Magnet Cold Mass for the Superconducting Super Collider*
}

\author{
K. K. Leung \\ Magnet Division \\ Superconducting Super Collider Laboratory ${ }^{\dagger}$ \\ 2550 Beckleymeade Ave. \\ Dallas, TX 75237 \\ T. Nicol \\ Fermi National Accelerator Laboratory $\ddagger$ \\ P. O. Box 500 \\ Batavia, IL 60510
}

March 1991

\footnotetext{
*Presented at the 1991 International Industrial Symposium on the Super Collider, Atlanta, Georgia, March 13-15, 1991.

tOperated by the Universities Research Association, Inc., for the U.S. Department of Energy under Contract No. DE-AC02-89ER40486.
}

†This work supported by the U.S. Department of Energy under Contract No. DE-AC02-76CH03000. 


\title{
A FREQUENCY RESPONSE STUDY OF DIPOLE MAGNET COLD MASS FOR THE SUPERCONDUCTING SUPER COLLIDER
}

\author{
K. K. Leung \\ Magnet Division \\ Superconducting Super Collider Laboratory* \\ 2550 Beckleymeade Avenue \\ Dallas, TX 75237 \\ T. Nicol \\ Fermi National Accelerator Laboratory ${ }^{\dagger}$ \\ P.O. Box 500 \\ Batavia, IL 60510
}

\begin{abstract}
This paper describes the technique for calculating the dynamic response of the Superconducting Super Collider (SSC) dipole magnet cold mass. Dynamic motion specification and beam location stability of the cold mass are not available at the present time. Dynamic response of the cold mass depends on measures excitation at the location of the magnet anchoring points and on other factors such as: (1) composite damping of the dipole magnet system, and (2) coupling effect of the cryogenic vessel, concrete slab, and soil to structure interactions. Nevertheless, the cold mass has the largest effect on the motion of the SSC machine.

This dynamic analysis is based on response spectra analysis using the finite element method. An upper bond solution will result from this method of analysis, compared to the transient dynamic response method which involves step-by-step time integration from recorded accelerograms. Since no recorded ground motions are available for the SSC site, response spectra from another source $^{1}$ shall be employed for the present analysis.
\end{abstract}

\section{INT RODUCTION}

Three-dimensional modal dynamic analysis is applied for evaluating the motion of the dipole cold mass. Maximum response is calculated from the combined individual response maxima of each mode. Dynamic modeling required selection of the correct dynamic degree of freedom in the cold mass to represent all significant modes. Compliance of the yokes, coils, and supporting posts is incorporated in the cold mass mathematical model. The experimental *Operated by the Universities Reasearch Association. Inc., for the U.S. Department of Energy under Contract
No. DE-AC02-89ER40486

TThis work supported by the U.S. Department of Energy under Contract No. DE-AC02-76CH03000. 
spring constants of the yokes and supporting posts will be the major dynamic characteristics in the present model. Similar dynamic motion studies of accelerator magnets for the U.S. Department of Energy have been performed. ${ }^{2}$ Experimental verification of a similar complex, three-dimensional model has been reported. ${ }^{3}$

The major structural element in controlling the cold mass rigidity is the dipole magnet skin, which also functions to: (1) perform as a pressure vessel, (2) provide an axial and radial direction pre-loads to the collared coil, and (3) serve as a rigid platform to integrate the coil and yoke assemblies.

The compliances for the yoke and the collared coil assembly, including gaps between each part, are shown in detail in Reference 4. The supporting post is a complex construction, with composite tubes and annular plates. The design of the supporting posts maximizes the heat conduction path with telescoping tubes and heat shield by the annular plates. The axial and lateral compliance is, therefore, non-linear and difficult to model analytically. T. Nicol of Fermi National Accelerator Laboratory (FNAL) has provided preliminary data on the supporting posts for the present analysis. Thermal restraint coupling of the dipole cold mass to the cryogenic vessel is avoided by de-coupling the axial thermal motion of the cold mass skin to all the supporting posts except the centering post. The structural coupling between the posts and the cold mass skin in the axial direction is, therefore, free for four of the posts.

\section{3-D FINITE ELEMENT MODEL}

A description of the finite element cold mass assembly is shown in Figure 1. The analysis was performed using ANSYS code $(4.4 \mathrm{a}+)$ on a VAX 6420 in the SSC Laboratory. The skin, yoke, end plate, vessel head, and supporting posts were modeled with plate and elastic pipe elements. The interface of the yoke to the skin is considered as integrated with the assumptions of low-level dynamic excitation, which will not exceed the frictional force between the yoke-to-skin interfaces. The body yoke was modeled with low axial elastic modulus to account for yoke laminations. The coil assembly is modeled with low elastic modulus to account for the Lorentz force on pre-loaded end plates. The eight-node stress solid element (stiff 73) is used for the yoke and coil assemblies. The cold mass skin is modeled with quad, flat-shell element (stiff 63). Elastic pipe element (stiff 16) is employed for the supporting posts, with axial and lateral compliances adjusted by the material property. There are 9030 nodes and 1565 elements with 15 total master dynamic degree of freedom in the 3-D model.

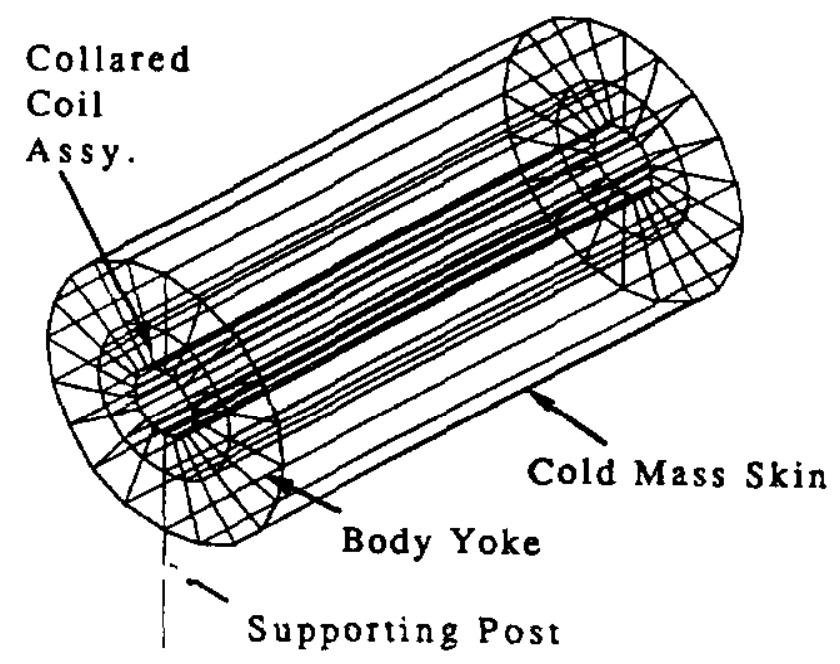

Figure 1. Dynamic Model of the Cold Mass Assembly. 
DYNAMIC EXCITATION APPLIED TO SSCL COLD MASS

Greens ${ }^{1}$ data is used as the dynamic excitation for the SSC cold mass response analysis. Figure 2 shows the ground response spectrum.

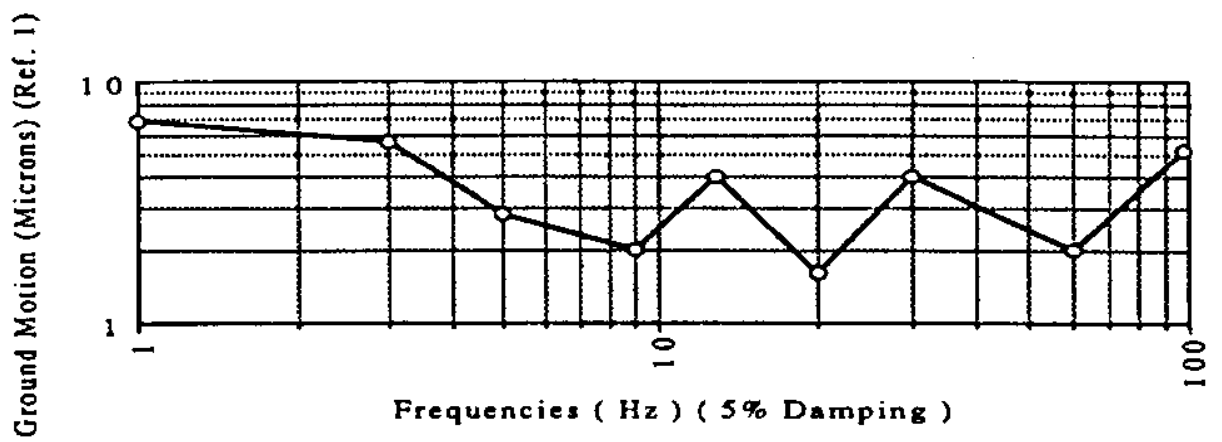

Figure 2. Ground Response Spectra for SSC Dipole Cold Mass Dynamic Study.

\section{RESULT OF DYNAMIC RESPONSE ANALYSIS}

Figure 3 shows that maximum modal deflection $=25$ microns of the collared coil assembly with three sigma probability $(99.75 \%)$.

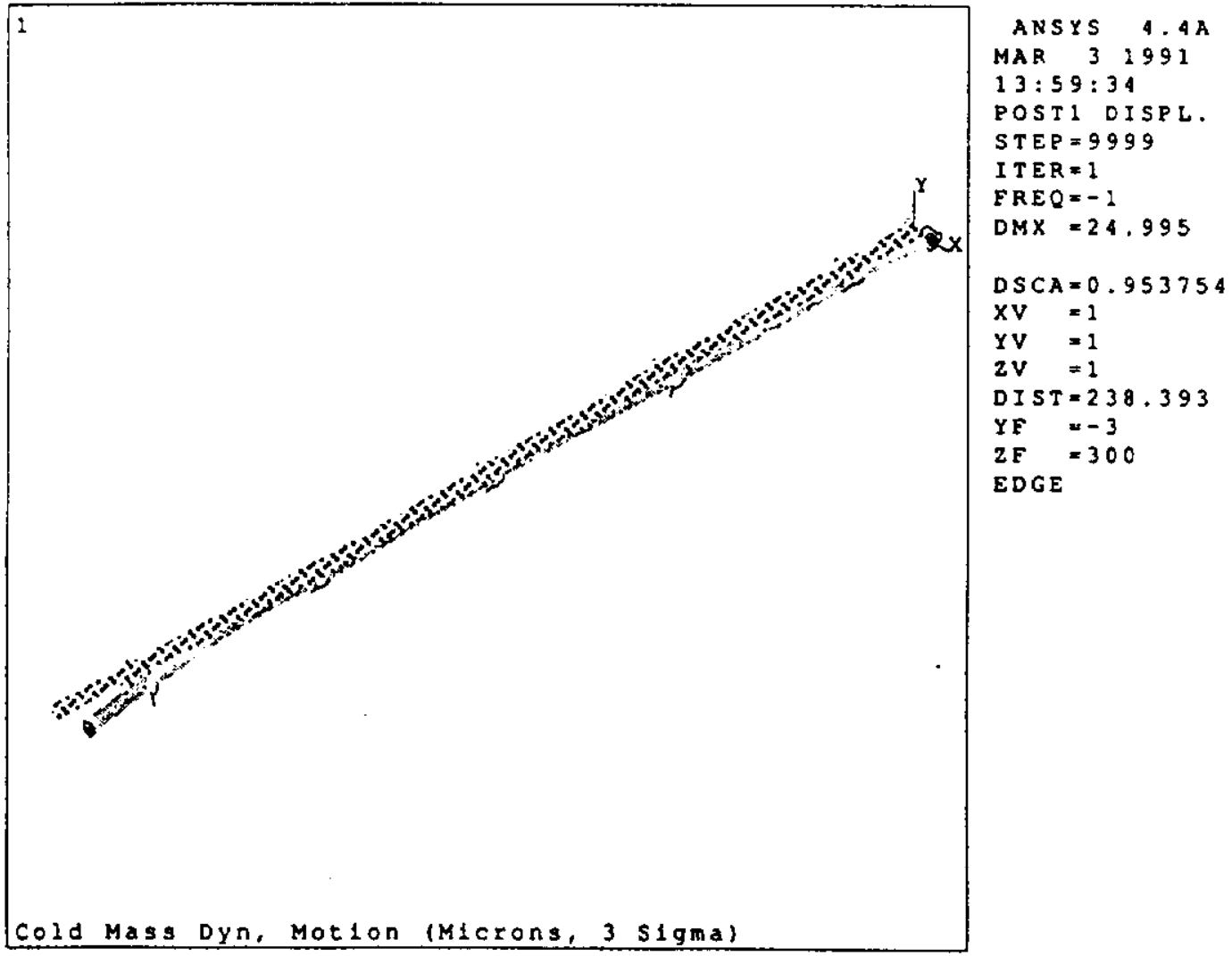

Figure 3. Modal Sum of Collared Coil Assembly. Lateral Deflection = 25 Microns (Max.). 


\section{RESULTS OF THE MODE-FREQUENCY ANALYSIS}

The most significant mode of the cold mass as calculated is $10.6 \mathrm{~Hz}$, which is indicated in Table 1 . The mode will have $58.63 \%$ of the cold mass weight moving laterally in response to the lateral ground excitation.

The analytical result is considered to be in good agreement with the $9.5 \mathrm{~Hz}$ experimental results as obtained at FNAL. The maximum lateral dynamic deflection is about 25 microns, which is obtained by a modal sum method called the square root of the sum of the squares approach. An amplification factor of $25 / 7=3.57$, based upon $5 \%$ damping assumption, is calculated from the present dynamic analysis.

The second significant mode of the cold mass is $4.9 \mathrm{~Hz}$, which has $37.5 \%$ of the effective mass moving in this mode. It must be pointed out that only lateral excitation is applied for the present analysis. Vertical excitation as well as axial excitation will be encountered by the cold mass system. The total response of the cold mass or the acceleration system can be evaluated by an integrated model with the SSCL site-specific response spectra in the future. The present analysis is done to demonstrate a simple and conservative technique to produce dynamic motion by using the finite element method along with the experimental compliances for the yoke, coil, and post.

Table 1. Summary For Dipole Cold Mass System Response Spectrum Calculation.

\begin{tabular}{cccccccc}
\hline \hline Mode & Freq. & SV & P. Factor & Mode Coef. M.C. Ratio & Erf. Mass & Mass Frac. \\
\hline 1 & 4.914 & 0.28663 & 6.122 & 1.755 & 0.842303 & 37.4786 & 0.339342 \\
2 & 5.773 & 0.25789 & $0.24 \mathrm{E}-02$ & $0.63 \mathrm{E}-03$ & 0.000306 & $0.609758 \mathrm{E}-05$ & 0.339342 \\
3 & 10.60 & 0.27208 & 7.657 & 2.083 & 1.000000 & 58.6293 & 0.870188 \\
4 & 13.87 & 0.34829 & $-0.24 \mathrm{E}-01$ & $-0.84 \mathrm{E}-02$ & 0.004060 & $0.589890 \mathrm{E}-03$ & 0.870193 \\
5 & 15.06 & 0.29261 & -1.433 & -0.4192 & 0.201218 & 2.05242 & 0.888776 \\
6 & 29.22 & 0.37678 & 2.154 & 0.8117 & 0.389620 & 4.64084 & 0.930796 \\
7 & 32.19 & 0.37275 & $-0.14 \mathrm{E}-01$ & $-0.52 \mathrm{E}-02$ & 0.002521 & $0.198546 \mathrm{E}-03$ & 0.930797 \\
8 & 46.42 & 0.25852 & $-0.58 \mathrm{E}-01$ & $-0.15 \mathrm{E}-01$ & 0.007243 & $0.340705 \mathrm{E}-02$ & 0.930828 \\
9 & 50.24 & 0.23884 & -0.4723 & -0.1128 & 0.054147 & 0.223063 & 0.932848 \\
10 & 53.87 & 0.22275 & 1.358 & 0.3024 & 0.145176 & 1.84358 & 0.949540 \\
11 & 85.89 & 0.40219 & 1.493 & 0.6004 & 0.288175 & 2.22821 & 0.969715 \\
12 & 128.0 & 0.52000 & 1.829 & 0.9510 & 0.456499 & 3.34481 & 1.00000 \\
13 & 195.0 & 0.52000 & $0.18 \mathrm{E}-03$ & $0.94 \mathrm{E}-04$ & 0.000045 & $0.327032 \mathrm{E}-07$ & 1.00000 \\
14 & 208.5 & 0.52000 & $-0.15 \mathrm{E}-02$ & $-0.78 \mathrm{E}-03$ & 0.000375 & $0.225258 \mathrm{E}-05$ & 1.00000 \\
15 & 240.3 & 0.52000 & $-0.13 \mathrm{E}-02$ & $-0.71 \mathrm{E}-03$ & 0.000345 & $0.191099 \mathrm{E}-05$ & 1.00000 \\
\hline \hline
\end{tabular}

Sum of Effective Masses $=110.445$

\section{DISCUSSIONS AND RECOMMENDATION}

1. In a well-damped system for the SSC, maximum dynamic deflection of the cold mass system will be less than 25 microns and may be as low as 2.5 microns. 
2. Site-specific response spectra need to be established.

3. Damping values and maximum allowable dynamic deflection for the cold mass system need to be established.

\section{REFERENCES}

1. M.A. Green et al., "Ground Motion Measurements at the LBL Light Source Site, the Bevatron and at SLAC," Lawrence Berkeley Laboratory Report LBL-21519, December 1986.

2. K.K. Leung, "Dynamic Motions Study of a Electron Storage Ring for the Third generation of Synchrotron Radiation Facility," Lawrence Berkeley Laboratory Report LSME-169, April 1989.

3. K.K. Leung, "Seismic Stress of the Piping Systems and Equipment on Heat exchanger Supporting Structures," Section K-7/6, Transactions of the 3rd International Conference on Structural Mechanics in Reactor Technology, London, United Kingdom, (1975).

4. J. Jayakumar et al., "Mechanical and Electromagnetic Analysis of a 50-mm Design for the SSC Dipole," Applied Superconductivity Conference, Snowmass, CO, September 1990. 